\title{
ISOMETRY GROUPS OF RIEMANNIAN MANIFOLDS WITH BOUNDED NORMS
}

\author{
SEONG-Hun PAENG
}

(Received May, 6, 2004, revised September 29, 2005)

\begin{abstract}
We generalize several results on the order of the isometry group of a compact manifold with negative Ricci curvature proved by Dai et al. under the assumption of bounded norm and an integral curvature bound. We also show that there exists a bound on the order of the isometry group depending on the weak norm of $M$.
\end{abstract}

1. Introduction. Let $M$ be an $n$-dimensional compact Riemannian manifold. If the Ricci curvature $\operatorname{Ric}_{M}$ of $M$ satisfies $\operatorname{Ric}_{M}<0$, then the isometry group $\operatorname{Isom}(M)$ of $M$ is finite by a classical theorem of Bochner (see [10]). Yamaguchi [12] found a bound on the order of isometry groups depending on the volume under the assumption of negative sectional curvatures. In [3], Dai et al. showed that if $\left\{M_{j}\right\}$ is a $C^{1, \alpha}$-convergent sequence of manifolds satisfying $-K \leq \operatorname{Ric}_{M_{j}} \leq-k<0$, the injectivity radius $\operatorname{inj}_{M_{j}} \geq i_{0}$ and the $\operatorname{volume} \operatorname{vol}\left(M_{j}\right) \leq V$, then the order of isometry groups satisfies $\varlimsup_{j \rightarrow \infty}\left|\operatorname{Isom}\left(M_{j}\right)\right| \leq\left|\operatorname{Isom}\left(M_{0}\right)\right|<\infty$, where $|S|$ denotes the cardinality of a set $S$. As a corollary, $|\operatorname{Isom}(M)| \leq N\left(n, K, k, i_{0}, V\right)$ for a constant $N$ depending only on $n, K, k, i_{0}, V$. In [11], Rong proved that a compact manifold with negative Ricci curvature admits no non-trivial invariant F-structure, which implies that if the sectional curvature $\left|K_{M}\right| \leq 1, \operatorname{Ric}_{M} \leq-K<0$ and the diameter $\operatorname{diam}(M) \leq d$, then $|\operatorname{Isom}(M)| \leq N_{1}(n, K, d)$. Although this theorem has no assumption on the lower bound of the injectivity radius, a lower bound of it is obtained from the main theorem of [11].

Petersen introduced the (weak) norm of a manifold in [10]. The above condition that $-K \leq \operatorname{Ric}_{M} \leq-k<0$ and $\operatorname{inj}_{M} \geq i_{0}$ gives a bound on the harmonic $C^{1, \alpha}$-norm. We recall briefly the definition of the (weak) norm of an $n$-dimensional Riemannian manifold $(M, g)$ on scale $r>0$, where $g$ is the metric of $M$ in $[9,10]$.

DEFINITION 1 [9]. The $C^{k, \alpha}$-norm of an $n$-dimensional Riemannian manifold $(M, g)$ on scale $r>0,\|(M, g)\|_{C^{k, \alpha}, r}$, is defined to be the infimum of positive numbers $Q$ such that there exist imbeddings

$$
\Phi_{\tau}: B(0, r) \subset \boldsymbol{R}^{n} \rightarrow U_{\tau} \subset M
$$

with images $U_{\tau}, \tau \in I$ (an index set), with the following properties:

(1) $e^{-2 Q_{\delta}} \leq \Phi_{\tau}^{*}(g) \leq e^{2 Q_{\delta}}$, where $\delta$ is the Euclidean metric;

(2) every metric ball $B\left(p, r e^{-Q} / 10\right)$ for $p \in M$ lies in some $U_{\tau}$;

(3) $\quad r^{|l|+\alpha}\left\|\partial^{l} g_{\tau, i j}\right\|_{C^{0, \alpha}} \leq Q$ for all multi-indices $l$ with $0 \leq|l| \leq k$, where $g_{\tau}=\Phi_{\tau}^{*} g$.

2000 Mathematics Subject Classification. 53C20.

Key words and phrases. Weak norm, killing vector field, isometry group. 
The weak norm $\|(M, g)\|_{C^{k, \alpha}, r}^{\mathrm{W}}$ is defined in a similar way except that $\Phi_{\tau}$ is assumed to be a local diffeomorphism instead of a diffeomorphism. Then we may regard $\Phi_{\tau}$ as a weak coordinate. If the (weak) coordinate charts are harmonic, we call such a norm a (weak) harmonic norm. In [8], it is proved that if the weak $C^{0, \alpha}$-norm is bounded and the diameter is sufficiently small, then $M$ is diffeomorphic to a nilmanifold up to finite cover. Instead of $C^{k, \alpha}$-norm, $L^{k, p}$-norm can be used.

For constants $k \geq 0, \alpha \in(0,1], r>0$ and a positive function $Q(r)$ with $\lim _{r \rightarrow 0} Q(r)=$ 0 , we define the following classes of $n$-dimensional complete Riemannian manifolds:

$$
\begin{aligned}
& \mathcal{M}^{k, \alpha}(n, Q)=\left\{(M, g) \mid\|(M, g)\|_{C^{k, \alpha}, r}^{\mathrm{W}} \leq Q(r)\right\}, \\
& \tilde{\mathcal{M}}^{k, \alpha}(n, Q)=\left\{(M, g) \mid\|(M, g)\|_{C^{k, \alpha}, r} \leq Q(r)\right\}, \\
& \mathcal{M}_{L}^{k, p}(n, Q)=\left\{(M, g) \mid\|(M, g)\|_{L^{k, p}, r}^{\mathrm{W}} \leq Q(r)\right\}, \\
& \tilde{\mathcal{M}}_{L}^{k, p}(n, Q)=\left\{(M, g) \mid\|(M, g)\|_{L^{k, p}, r} \leq Q(r)\right\} .
\end{aligned}
$$

Note that $\mathcal{M}_{L}^{k+1, p}(n, Q) \subset \mathcal{M}^{k, \alpha}(n, Q)$.

For given $Q, n, k, r>0, \tilde{\mathcal{M}}^{k, \alpha}(n, Q)$ is compact in the pointed $C^{k, \alpha^{\prime}}$-topology for any $\alpha^{\prime}<\alpha$ (see [11]). Furthermore, Petersen et al. showed that if $M \in \mathcal{M}^{0, \alpha}(n, Q)$ for a harmonic weak coordinate chart, then the metric $g$ of $M$ can be deformed to a metric $g^{\prime}$ with $\left|K_{g^{\prime}}\right| \leq K$, where $K_{g^{\prime}}$ is the sectional curvature with respect to the metric $g^{\prime}$ and $K$ depends on $\alpha, Q$ (see [9]). So the (weak) norm can be considered as a generalization of the curvature.

It is our question if we can generalize the above results on the isometry group of a negatively curved manifold under a bounded (weak) norm. It should be noted that it has not been known that manifolds in $\mathcal{M}^{1, \alpha}(n, Q)$ can be deformed to those with metrics of bounded sectional curvature without harmonicity. So we cannot use the arguments on F-structure in [12] and it is not known whether collapsing could occur.

We will prove the following theorems. We can easily generalize the result in [3] under an integral bound on Ricci curvature as follows: let $h(x)$ be the largest eigenvalue for the Ricci transformation Ric : $T_{x} M \rightarrow T_{x} M$. Similarly as in [10], we consider

$$
\overline{\mathcal{R}}_{M}(K)=\frac{1}{\operatorname{vol}(M)} \int_{M} \max \{h(x)+K, 0\} d v .
$$

The classical Bochner theorem claims that if $\overline{\mathcal{R}}_{M}(K)=0$ for some $K>0$, then $|\operatorname{Isom}(M)|<\infty$ (see [11]). First, as a generalization of [3], we prove the following.

THEOREM 1. For fixed $K, p>0$ and a positive function $Q(r)$ with $\lim _{r \rightarrow 0} Q(r)=0$, let $M_{j}$ be a sequence of manifolds in $\tilde{\mathcal{M}}_{L}^{2, p}(n, Q)$ satisfying $\operatorname{vol}\left(M_{j}\right) \leq V$. If $M_{j}$ converges to $M$ in the $L^{2, p}$-topology and $\overline{\mathcal{R}}_{M_{j}}(K) \rightarrow 0$, then:

(1) $|\operatorname{Isom}(M)|<\infty$; and

(2) $\varlimsup_{i \rightarrow \infty}\left|\operatorname{Isom}\left(M_{j}\right)\right| \leq|\operatorname{Isom}(M)|$.

COROLlaRY 1. For a given $K>0$ and $M \in \tilde{\mathcal{M}}_{L}^{2, p}(n, Q)$ satisfying $\operatorname{vol}(M) \leq$ $V$, there exist constants $N(n, K, Q, p, V)$ and $\varepsilon(n, K, Q, p, V)>0$ depending only on 
$n, K, Q, p, V$ such that if $\overline{\mathcal{R}}_{M}(K)<\varepsilon(n, K, Q, p, V)$, then the order of the isometry group is bounded by $N(n, K, Q, p, V)$.

Note that the class of manifolds satisfying $-k \leq \operatorname{Ric}_{M} \leq-K$ for some constants $k, K>$ $0, \operatorname{inj}_{M} \geq i_{0}$ and $\operatorname{vol}(M) \leq V$ is contained in $\tilde{\mathcal{M}}_{L}^{2, p}(n, Q)$ for some $p, Q, r$ by [1]. Our proof is much simpler than that of [3]. Corollary 1 can be considered as an integral version of the Bochner theorem.

It is not known whether Theorem 1 can be generalized to the class $\mathcal{M}^{1, \alpha}(n, Q)$ (or $\left.\mathcal{M}_{L}^{2, p}(n, Q)\right)$ as collapsing could occur. However, our main result is to prove the corollary without non-collapsing conditions (e.g., lower bound on the injectivity radius) as follows.

THEOREM 2. Let $K, Q, \alpha$ be positive numbers and $Q(r)$ be a positive function satisfying $\lim _{r \rightarrow 0} Q(r)=0$. For $M \in \mathcal{M}^{1, \alpha}(n, Q)$ with $\operatorname{diam}(M) \leq d$, there exist constants $\varepsilon(n, K, Q, \alpha, d)>0$ and $N(n, K, Q, \alpha, d)$ depending only on $n, K, Q, \alpha, d$ such that if $\overline{\mathcal{R}}(K)<\varepsilon(n, K, Q, \alpha, d)$, then the order of the isometry group is bounded by $N(n, K, Q, \alpha, d)$.

It can be considered as a generalization of a result in [11]. In [11], Rong showed that a collapsing does not occur from the main result and used the theorem in [3]. However, in our case, we do not assume that collapsing does not occur, so that we cannot obtain the compactness theorem under a bound of the weak $C^{k, \alpha}$-norm.

2. Proof of Theorem 1. We prove the following theorem which has been proved in [3, Theorem 1.3] under the assumption of bounded Ricci curvature. Once we prove the theorem, the remaining part of the proof of Theorem 1 and Corollary 1 is the same as those in [3], which requires only $L^{2, p}$-convergence of $M_{j}$.

THEOREM 3. Let Mbe an n-dimensional compact Riemannian manifold in $\tilde{\mathcal{M}}_{L}^{2, p}(n, Q)$ with $\lim _{r \rightarrow 0} Q(r)=0$ and $\operatorname{vol}(M) \leq V$. Then there exists $\varepsilon(n, K, Q, p, V)>0$ such that if $\overline{\mathcal{R}}_{M}(K)<\varepsilon(n, K, Q, p, V)$ and $\max \{d(\phi(x), x) \mid x \in M\} \leq \varepsilon(n, K, Q, p, V)$ for an isometry $\phi$, then $\phi$ is the identity map.

ProOF. Let $\left\{M_{j} \mid j=1,2, \ldots\right\}$ be a sequence of manifolds in $\tilde{\mathcal{M}}_{L}^{2, p}(n, Q)$ such that $\overline{\mathcal{R}}_{M_{j}} \rightarrow 0, \operatorname{vol}\left(M_{j}\right) \leq V$ and $\operatorname{diam}\left(M_{j}\right) \leq D$ for a positive constant $D>0$ and any $j>0$. Assume that $M_{j}$ has an isometry $\phi_{j}$ such that $\max \left\{d\left(\phi_{j}(x), x\right) \mid x \in M_{j}\right\}=\varepsilon_{j} \rightarrow 0$. We denote by $(X, p)_{g}$ a manifold $X$ pointed at $p$ with a metric $g$. We choose $p_{j} \in M_{j}$ such that $d\left(\phi_{j}\left(p_{j}\right), p_{j}\right) \geq \varepsilon_{j} / 2$. Rescaling the metric $g_{j}$ of $M_{j}$ by multiplying $\varepsilon_{j}^{-2}$, we have the following convergence for $p_{j} \in M_{j}$ :

$$
\left(M_{j}, p_{j}\right)_{\varepsilon_{j}^{-2} g_{j}} \rightarrow\left(\boldsymbol{R}^{n}, o\right)_{\delta}
$$

in the $C^{1, \alpha}$-topology and $\phi_{j} \rightarrow \phi \in \operatorname{Isom}\left(\boldsymbol{R}^{n}\right)$ in the $C^{2}$-topology from the proof of [2], where $\phi(x)=A x+b$ for $A \in O\left(n, \boldsymbol{R}^{n}\right)$ and $b \neq 0 \in \boldsymbol{R}^{n}$ and $d(\phi(x), x) \leq 1$ for all $x \in \boldsymbol{R}^{n}$. Then it follows that $A=I$ and $\phi_{j}$ is almost translational. As $(R x+t)^{k}=R^{k} x+\left(R^{k-1}+\cdots+I\right) t$ on $\boldsymbol{R}^{n}$, if $\phi_{j_{*}} \rightarrow I$, then the order of $\phi_{j}$ satisfies that $\left|\phi_{j}\right| \rightarrow \infty$. 
Considering a subsequence, we may assume that $\left(M_{j},\left\langle\phi_{j}\right\rangle\right) \rightarrow\left(M_{0}, Z\right)$ with respect to the equivalent Hausdorff distance for some $C^{1, \alpha}$-manifold $M_{0}$ and an isometry group $Z$ on $M_{0}$. There exists a diffeomorphism $F_{j}: M_{0} \rightarrow M_{j}$ such that $F_{j}^{*} g_{j} \rightarrow g_{0}$ in the $C^{1, \alpha}-$ topology. First, we show that $Z$ has a non-trivial element. If $Z$ has no non-trivial element, then the diameter of the orbit of $\phi_{j} \operatorname{diam}\left(\left\{\phi_{j}^{k}(x) \mid k \in Z\right\}\right) \rightarrow 0$ for every $x \in M_{j}$. Let $\varepsilon_{j}$ be $\sup _{x \in M_{j}} \operatorname{diam}\left(\left\{\phi_{j}^{k}(x) \mid k \in Z\right\}\right)$ and we choose $p_{j} \in M_{j}$ such that $\operatorname{diam}\left(\left\{\phi_{j}^{k}\left(p_{j}\right) \mid k \in \boldsymbol{Z}\right\}\right) \geq$ $\varepsilon_{j} / 2$. Rescaling the metric $g_{j}$ of $M_{j}$ by multiplying $\varepsilon_{j}^{-2}$, we have the following convergence for $p_{j} \in M_{j}$ and $Z^{\prime} \subset \operatorname{Isom}\left(\boldsymbol{R}^{n}\right)$ :

$$
\left(M_{j}, p_{j},\left\langle\phi_{j}\right\rangle\right)_{\varepsilon_{j}^{-2} g_{j}} \rightarrow\left(\boldsymbol{R}^{n}, o, Z^{\prime}\right)_{\delta},
$$

where $\sup \left\{d(\phi(o), o) \mid \phi \in Z^{\prime}\right\} \leq 1$. For the precise definition of pointed Hausdorff approximation, see [4]. It follows from the above arguments that $\phi_{j}^{k}$ converges to a translational isometry as $j \rightarrow \infty$ for all $k$, since $d\left(\phi_{j}^{k}(x), x\right) \leq \varepsilon_{j}$. However, this leads to a contradiction as follows: if $d\left(\phi_{j}^{k_{0}}(x), x\right) \geq 3 \sup _{k} d\left(\phi_{j}^{k}(x), x\right) / 4$, then $d\left(\phi_{j}^{2 k_{0}}(x), x\right)>\sup _{k} d\left(\phi_{j}^{k}(x), x\right)$ for sufficiently large $j$, as $\phi_{j}^{k_{0}}$ is almost translational, which is a contradiction.

Now we show that $|Z|=\infty$. For $\gamma \in Z$, let $d(\gamma(p), p)=\beta>0$ and $\phi_{j}^{k_{j}} \rightarrow \gamma$. Then there exists $l_{j}>0$ such that $\beta / 3<d\left(\phi_{j}^{l_{j}}\left(p_{j}\right), p_{j}\right)<\beta / 2$, where $p_{j} \rightarrow p$. Considering a subsequence of $\left\{\phi_{j}^{l_{j}}\right\}$, we can construct an isometry $\gamma_{1}$ with $\beta / 3<d\left(\gamma_{1}(p), p\right)<\beta / 2$. Inductively, we can construct isometries $\gamma_{m}$ for each positive integer $m$ such that $\beta /(m+2)<d\left(\gamma_{m}(p), p\right)<\beta /(m+1)$. Hence, $|Z|=\infty$.

As $|Z|=\infty$, there exists a non-trivial Killing vector field $X$ on $M_{0}$. Let $A$ be $\max _{x \in M_{0}}\|X(x)\|>0$. It is well known that if $Y$ is a compact Riemannian manifold, then $\operatorname{Isom}(Y)$ is a Lie group and there exists a Killing vector field when $|\operatorname{Isom}(Y)|=\infty$ (see [7]). Let $X_{j}$ be a vector field on $M_{j}$ defined by $F_{j_{*}} X$. Then by Bochner's formula (see [3]), we have

$$
\int_{M_{j}}\left\{\operatorname{Ric}_{M_{j}}\left(X_{j}, X_{j}\right)+(1 / 2)\left|L_{X_{j}} g_{j}\right|^{2}-\left|\nabla^{g_{j}} X_{j}\right|^{2}-\left(\operatorname{div}^{g_{j}} X_{j}\right)^{2}\right\} d v=0 .
$$

Note that $M_{j}$ can be identified with $M_{0}$ by $F_{j}$ as a space with different metrics. Then $X_{j}$ and $X$ also can be considered as the same vector field on the same space $M_{0}=M_{j}$. In general, we have that

$$
\begin{aligned}
2 g\left(\nabla_{X}^{g} Y, Z\right)= & X(g(Y, Z))+Y(g(Z, X))-Z(g(X, Y)) \\
& +g([X, Y], Z)-g([X, Z], Y)-g([Y, Z], X) .
\end{aligned}
$$

As $F_{j}^{*} g_{j} \rightarrow g_{0}$ in the $C^{1, \alpha}$-topology and $F_{j *} X=X_{j}$ (i.e., $X_{j}=X$ if we identify $M_{j}$ with $M_{0}$ by $F_{j}$ ), it follows from (3) that

$$
\begin{aligned}
\left(L_{X_{j}} g_{j}\right)(V, W) & =g_{j}\left(\nabla_{V}^{g_{j}} X_{j}, W\right)+g_{j}\left(\nabla_{W}^{g_{j}} X_{j}, V\right) \\
& \rightarrow g_{0}\left(\nabla_{V}^{g_{0}} X, W\right)+g_{0}\left(\nabla_{W}^{g_{0}} X, V\right)=\left(L_{X} g_{0}\right)(V, W)=0
\end{aligned}
$$


as $j \rightarrow \infty$. Let $S_{j}$ be $\{x \mid h(x)+K>0\}$. As

$$
\begin{aligned}
\int_{M_{j}} & \operatorname{Ric}_{M_{j}}\left(X_{j}, X_{j}\right) d v+\int_{M_{j}} K\left\|X_{j}\right\|^{2} d v \\
& \leq \int_{M_{j}}(h(x)+K)\left\|X_{j}\right\|^{2} d v \\
& \leq \int_{S_{j}}(h(x)+K)\left\|X_{j}\right\|^{2} d v \leq 2 A^{2} \overline{\mathcal{R}}_{M_{j}}(K) \operatorname{vol}\left(M_{j}\right) \rightarrow 0
\end{aligned}
$$

and

$$
\int_{M_{j}}\left\{\left|\nabla^{g_{j}} X_{j}\right|^{2}+\left(\operatorname{div}^{g_{j}} X_{j}\right)^{2}\right\} d v \geq 0
$$

as $j \rightarrow \infty$, we obtain $X=0$, which is a contradiction.

3. Proof of Theorem 2. Under the condition in Theorem 2, we cannot use $C^{1, \alpha}$ compactness theorem, so that we cannot obtain an almost Killing vector field in the limit space with $C^{1, \alpha}$-metric directly. Hence, we first construct local Killing vector fields and then paste them together. We are going to prove Theorem 2 by contradiction. Assume that $\left\{M_{j}\right\}$ is a sequence of manifolds satisfying conditions in Theorem 2 and $\overline{\mathcal{R}}_{M_{j}}(K) \rightarrow 0$ but $\left|\operatorname{Isom}\left(M_{j}\right)\right| \rightarrow \infty$.

Fix a sufficiently small $r>0$ such that an $r$-ball in $M \in \tilde{\mathcal{M}}_{L}^{2, p}(n, Q)$ can be considered as an almost flat structure [8] and $Q(r)<1 / 100$. Let $\phi$ be an isometry on $M$ such that $d(\phi(p), p)<r / 10000$. Then $\phi$ can be lifted to an isometry $\tilde{\phi}: B(0, r / 100)_{\Phi^{*}\left(g_{j}\right)} \rightarrow$ $B(0, r / 2)_{\Phi^{*}\left(g_{j}\right)}$ for a weak coordinate $\Phi$ with $\Phi(0)=p$, where $B(0, s)_{\Phi^{*}\left(g_{j}\right)}$ for $s<r$ is the set $\left\{\left(x_{1}, x_{2}, \ldots, x_{n}\right) \in \boldsymbol{R}^{n} \mid \sum_{i} x_{i}^{2}=s^{2}\right\}$ with a metric $\Phi^{*}\left(g_{j}\right)$. A pseudogroup means a set $\Gamma$ with a product $\alpha \beta \in \Gamma$ defined for some $\alpha, \beta \in \Gamma$ and the local fundamental pseudogroup is the set of geodesic loops based at a given point whose lengths are smaller than a positive constant. (For the precise definition of pseudogroup and local fundamental pseudogroup, see [5].) Let $\Gamma_{p}$ be the local fundamental pseudogroup of geodesic loops in $B(p, r / 100)$ based at $p$ whose lengths are smaller than $r / 100$. Then elements of $\Gamma_{p}$ can be considered as isometric embeddings from $B(0, r / 100)_{\Phi^{*}\left(g_{j}\right)}$ into $B(0, r / 2)_{\Phi^{*}\left(g_{j}\right)}$.

We may assume that $M_{j} \rightarrow M_{0}$ for a compact length space $M_{0}$ with respect to the Gromov-Hausdorff distance. From the compactness of $M_{0}$, we can find a $C_{0}$-covering $\left\{B\left(p_{k}^{j}, r / 100\right) \mid p_{k}^{j} \in M_{j}, k=1, \ldots, C_{0}\right\}$ of $M_{j}$ such that $\left(\Phi_{k}^{j}\right)^{*} g_{j} \rightarrow g^{k}$ in the $C^{1, \alpha}$-norm, where $\Phi_{k}^{j}: B(0, r / 100) \subset \boldsymbol{R}^{n} \rightarrow M_{j}$ are weak coordinates around $p_{k}^{j}$. For each $k$, we have the convergence

$$
\left(B(0, r / 100), \Gamma_{p_{k}^{j}}\right)_{\left(\Phi_{k}^{j}\right)^{*} g_{j}} \rightarrow\left(\boldsymbol{B}_{k}, \boldsymbol{\Gamma}_{k}\right)_{\boldsymbol{g}^{k}},
$$

and there exists a diffeomorphism $F_{k}^{j}: \boldsymbol{B}_{k} \rightarrow B(0, r / 100)$. For convenience, we abbreviate $\left(\Phi_{k}^{j}\right)^{*} g_{j}$ to $g_{j}$. We may identify $\boldsymbol{B}_{k}$ with $B(0, r / 100)$ as a space.

We prove Theorem 2 by a contradiction. Assume that there is a sequence of manifolds $\left\{M_{j}\right\}$ in $\mathcal{M}^{1, \alpha}(n, Q)$ such that there exists a sequence of isometries $\phi_{j}: M_{j} \rightarrow M_{j}$ with 
$\max \left\{d\left(\phi_{j}(x), x\right) \mid x \in M_{j}\right\} \rightarrow 0$. Then we have $\tilde{\phi}_{j} \circ \gamma \circ \tilde{\phi}_{j}^{-1} \in \Gamma_{p}$ for $\gamma \in \Gamma_{p}$ and

$$
d\left(\tilde{x}, \tilde{\phi}_{j}(\tilde{x})\right) \leq d(\tilde{x}, \tilde{p})+d\left(\tilde{p}, \tilde{\phi}_{j}(\tilde{p})\right)+d\left(\tilde{\phi}_{j}(\tilde{p}), \tilde{\phi}_{j}(\tilde{x})\right) \leq d\left(\tilde{p}, \tilde{\phi}_{j}(\tilde{p})\right)+r / 50
$$

for all $\tilde{x} \in B(0, r / 100)_{\Phi^{*}\left(g_{j}\right)}$.

For the proof of Theorem 2, we need the following lemma.

LEMMA 1. If $\left|\operatorname{Isom}\left(M_{j}\right)\right| \rightarrow \infty$, then there exists a sequence of isometries $\left\{\phi_{j}\right\}$ such that $\left(B(0, r / 100),\left\langle\phi_{j}\right\rangle\right)_{\left(\Phi_{k}^{j}\right)^{*} g_{j}} \rightarrow\left(\boldsymbol{B}_{k}, \phi_{t}\right)_{g^{k}}$ for a one-parameter isometry (pseudo)subgroup $\phi_{t}$, which yields a non-trivial Killing vector field $X_{k}=d \phi_{t} / d t$ on $\boldsymbol{B}_{k}$ for each $k$.

In our case, even if there exists a sequence of isometries $\phi_{j}: M_{j} \rightarrow M_{j}$ such that $\max _{x}\left\{d\left(\phi_{j}(x), x\right)\right\} \rightarrow 0$, it may occur that $\max _{x}\left\{d\left(\tilde{\phi}_{j}(\tilde{x}), \tilde{x}\right)\right\}$ does not converge to 0 , i.e., rotational parts do not necessarily converge to $I$ if $\left[\tilde{\phi}_{j}, \Gamma_{p_{j}}\right] \neq 0$, which is the main difference from the case of Theorem 1. (In the proof of Theorem 1, $\max \left\{d\left(\phi_{j}(x), x\right)\right\} \rightarrow 0$ implies that $\phi_{j}$ is almost translational.) We prove this lemma in Section 4. It follows from the proof of Lemma 1 that $\left\{\left\|X_{k}\right\|_{C^{1}} \mid k=1, \ldots\right\}$ is bounded.

As $L_{X_{k}} \boldsymbol{g}^{k}=0$, we have that $\boldsymbol{g}^{k}\left(\nabla_{V} X_{k}, W\right)+\boldsymbol{g}^{k}\left(\nabla_{W} X_{k}, V\right)=0$. Furthermore, on each $\boldsymbol{B}_{k}$, every $X_{k}$ can be obtained as a derivative of a one-parameter subgroup of isometries, which is the limit of one cyclic group of isometries on $M_{j}$. We identify $\boldsymbol{B}_{k}$ with $B(0, r / 100)$ by the diffeomorphism $F_{k}^{j}$. As $X_{k_{i}}$ are generated by one cyclic group of isometries on $M_{j}$, we have

$$
\left(\Phi_{k_{1}}^{j}\right)_{*}\left(X_{k_{1}}\left(x_{1}\right)\right)=\left(\Phi_{k_{2}}^{j}\right)_{*}\left(X_{k_{2}}\left(x_{2}\right)\right)
$$

for some $x_{1}, x_{2}$ such that $\Phi_{k_{1}}^{j}\left(x_{1}\right)=\Phi_{k_{2}}^{j}\left(x_{2}\right)$.

As $\tilde{\phi}_{j} \circ \gamma^{j} \circ \tilde{\phi}_{j}^{-1} \in \Gamma_{p_{k}^{j}}$ for $\gamma^{j} \in \Gamma_{p_{k}^{j}}$, we see that for $\gamma \in \boldsymbol{\Gamma}_{k}$

$$
\gamma_{*} X_{k}=X_{k}
$$

As $\gamma^{j} \rightarrow \gamma$ in the $C^{2}$-topology for $\gamma^{j} \in \Gamma_{p_{k}^{j}}$ and $\gamma \in \boldsymbol{\Gamma}_{k}$ from [2], we have

$$
\left\|\left(\Phi_{k}^{j}\right)_{*}\left(X_{k}(x)\right)-\left(\Phi_{k}^{j}\right)_{*}\left(X_{k}\left(\gamma^{j} x\right)\right)\right\|_{C^{1}} \rightarrow 0
$$

for $\gamma^{j} \in \Gamma_{p_{k}^{j}}$ from (7). Now we construct a global smooth vector field $\mathcal{X}^{j}$ on $M_{j}$ which is close to $\left(\Phi_{k}^{j}\right)_{*} X_{k}$ in the $C^{1}$-topology. Let $A_{k}^{j}$ be the set of pseudogroup defined by $A_{k}^{j}=$ $\left\{\gamma \in \Gamma_{p_{k}^{j}} \mid \gamma p_{k}^{j} \in B\left(p_{k}^{j}, r / 100\right)\right\}$. Let $X_{k}^{j}$ be a $C^{1}$-vector field on $\operatorname{Im}\left(\Phi_{k}^{j}\right)$ defined as follows:

$$
\boldsymbol{X}_{k}^{j}(x)=\frac{1}{\left|A_{k}^{j}\right|} \sum_{\gamma \in A_{k}^{j}}\left(\Phi_{k}^{j}\right)_{*}\left(X_{k}(\gamma \tilde{x})\right),
$$

where $\tilde{x}$ is a point in $\left(\Phi_{k}^{j}\right)^{-1}(x)$. Then from (8), (3) and $g_{j} \rightarrow g$ in $C^{1, \alpha}$, it follows that

$$
\begin{gathered}
\left\|\nabla_{V}^{g_{j}} \boldsymbol{X}_{k}^{j}-\nabla_{V}^{g_{j}}\left(\Phi_{k}^{j}\right)_{*}\left(X_{k}\right)\right\|_{C^{0}} \rightarrow 0, \\
\left\|g_{j}\left(\nabla_{V}^{g_{j}}\left(\Phi_{k}^{j}\right)_{*}\left(X_{k}\right), W\right)-\boldsymbol{g}^{k}\left(\nabla_{V}^{\boldsymbol{g}^{k}} X_{k}, W\right)\right\|_{C^{0}} \rightarrow 0
\end{gathered}
$$


almost everywhere for smooth vector fields $V$ and $W$ on $\operatorname{Im}\left(\Phi_{k}^{j}\right)$ as $j \rightarrow \infty$. Similarly as in (4), we obtain that

$$
L_{\boldsymbol{X}_{k}^{j}} g_{j} \rightarrow L_{X_{k}} g^{k}=0,
$$

as $\boldsymbol{X}_{k}^{j} \rightarrow X_{k}$ and $g_{j} \rightarrow \boldsymbol{g}^{k}$ in the $C^{1}$-topology, identifying $\boldsymbol{B}_{k}$ and $\operatorname{Im}\left(\Phi_{k}^{j}\right)$ locally. Using a partition of unity, we can construct a globally continuous vector field $\overline{\mathcal{X}}^{j}$ from $\boldsymbol{X}_{k}^{j}$. Let $\psi:[0, r) \rightarrow[0,1)$ be a decreasing smooth function such that $\psi(x)=1$ on $\left[0, r / 100-\varepsilon_{0}\right)$ and 0 for $x \geq r / 100$ for a fixed $\varepsilon_{0}<r / 10000$. If $\psi_{k}(x)=\psi\left(d\left(p_{k}^{j}, x\right)\right)$, then $\left\{\bar{\psi}_{k} \mid \bar{\psi}_{k}=\right.$ $\left.\psi_{k} /\left(\sum_{k} \psi_{k}\right)\right\}$ is a partition of unity. Let $\overline{\mathcal{X}}^{j}$ be $\sum_{k} \bar{\psi}_{k} \boldsymbol{X}_{k}^{j}$. As $\nabla \bar{\psi}_{k}$ is uniformly bounded almost everywhere, $\lim _{j \rightarrow \infty}\left|A_{k}^{j}\right|=\infty$ and

$$
\left\|\nabla_{V}^{g_{j}} \boldsymbol{X}_{k_{1}}^{j}-\nabla_{V}^{g_{j}} \boldsymbol{X}_{k_{2}}^{j}\right\|_{C^{0}} \rightarrow 0
$$

by (6) and (9), we have

$$
\begin{aligned}
\left\|\nabla_{V}^{g_{j}} \overline{\mathcal{X}}^{j}-\nabla_{V}^{g_{j}}\left(\Phi_{k}^{j}\right)_{*}\left(X_{k}\right)\right\|_{C^{0}}= & \left\|\nabla_{V}^{g_{j}} \sum_{k} \bar{\psi}_{k} \boldsymbol{X}_{k}^{j}-\nabla_{V}^{g_{j}}\left(\Phi_{k}^{j}\right)_{*}\left(X_{k}\right)\right\|_{C^{0}} \\
\leq & \left\|d\left(\sum_{k} \bar{\psi}_{k}\right)(V) \boldsymbol{X}_{k_{0}}^{j}+\sum_{k} d \bar{\psi}_{k}(V)\left(\boldsymbol{X}_{k}^{j}-\boldsymbol{X}_{k_{0}}^{j}\right)\right\|_{C^{0}} \\
& +\left\|\sum_{k} \bar{\psi}_{k} \nabla_{V}^{g_{j}} \boldsymbol{X}_{k}^{j}-\nabla_{V}^{g_{j}}\left(\Phi_{k}^{j}\right)_{*}\left(X_{k}\right)\right\|_{C^{0}} \rightarrow 0
\end{aligned}
$$

almost everywhere as $j \rightarrow \infty$. Note that $\sum_{k} \bar{\psi}_{k}=1$.

Now we follow the smoothing technique in [6]. Choose $\rho_{j}$ with $0<\rho_{j}<\operatorname{inj}_{M_{j}}$. Let $d \mu_{x}$ be the measure on $B\left(x, \rho_{j}\right)$ induced from the Lebesgue measure on $\left\{v \in T_{x} M_{j} \mid\|v\|<\right.$ $2 \rho_{j}$ \} by exp. We define the smoothing kernel $\Psi_{\rho_{j}}: M_{j} \times M_{j} \rightarrow \boldsymbol{R}$ by

$$
\Psi_{\rho_{j}}(x, y):=\frac{\psi\left(\rho_{j}^{-1} d(x, y)\right)}{\int_{B\left(x, \rho_{j}\right)} \psi\left(\rho_{j}^{-1} d(x, \cdot)\right) d \mu_{x}} .
$$

We denote by $P_{x}^{y}(V)$ the parallel translation of $V$ from $x$ to $y$ along the minimal geodesic.

Let $\mathcal{X}^{j}$ be defined as follows:

$$
\mathcal{X}^{j}(x)=\int_{B(x, \rho)} P_{y}^{x}\left(\overline{\mathcal{X}}^{j}(y)\right) \Psi_{\rho_{j}}(x, y) d \mu_{x} .
$$

For $u \in T_{x} M_{j}$, let $\gamma$ be the geodesic from $x$ with $\gamma^{\prime}(0)=u$. Also we let $\gamma_{y}(t)=$ $\exp _{\gamma(t)}\left(P_{x}^{\gamma(t)}\left(\exp _{\gamma(0)}^{-1}(y)\right)\right)$. Let $U$ be the vector field defined as $U(y)=\gamma_{y}^{\prime}(0)$. As $\exp _{\gamma(t)} \circ P_{x}^{\gamma(t)} \circ \exp _{\gamma(0)}^{-1}$ is measure preserving, we have

$$
\mathcal{X}^{j}(\gamma(t))=\int_{B(x, \rho)} P_{\gamma_{y}(t)}^{\gamma(t)}\left(\overline{\mathcal{X}}^{j}\left(\gamma_{y}(t)\right) \Psi_{\rho_{j}}(x, y) d \mu_{x} .\right.
$$


Note that

$$
\begin{aligned}
& \left\|P_{\gamma(t)}^{\gamma(0)} \circ P_{\gamma_{y}(t)}^{\gamma(t)}(X)-P_{\gamma_{y}(0)}^{\gamma(0)}(X)-P_{\gamma_{y}(0)}^{\gamma(0)} \circ\left(P_{\gamma_{y}(t)}^{\gamma_{y}(0)}(X)-X\right)\right\| \\
& \quad=\left\|P_{\gamma(t)}^{\gamma(0)} \circ P_{\gamma_{y}(t)}^{\gamma(t)}(X)-P_{\gamma_{y}(0)}^{\gamma(0)} \circ P_{\gamma_{y}(t)}^{\gamma_{y}(0)}(X)\right\| \leq K_{j} t \rho_{j}\|X\|,
\end{aligned}
$$

where $K_{j}$ depending on the sectional curvature $K_{M_{j}}$ of $M_{j}$. Hence,

$$
\begin{aligned}
& \| \frac{d}{d t} P_{\gamma_{y}(t)}^{\gamma(t)}\left(\left.\overline{\mathcal{X}}^{j}\left(\gamma_{y}(t)\right)\right|_{t=0}-P_{\gamma_{y}(0)}^{\gamma(0)}\left(\nabla_{U}^{g_{j}} \overline{\mathcal{X}}^{j}\right) \|\right. \\
& \quad=\left\|\lim _{t \rightarrow 0} \frac{P_{\gamma(t)}^{\gamma(0)} P_{\gamma_{y}(t)}^{\gamma(t)}\left(\overline{\mathcal{X}}^{j}\right)-P_{\gamma_{y}(0)}^{\gamma(0)}\left(\overline{\mathcal{X}}^{j}\right)}{t}-\frac{P_{\gamma_{y}(0)}^{\gamma(0)} \circ\left(P_{\gamma_{y}(t)}^{\gamma_{y}(0)}\left(\overline{\mathcal{X}}^{j}\right)-\overline{\mathcal{X}}^{j}\right)}{t}\right\| \\
& \quad \leq K_{j} \rho_{j}\left\|\overline{\mathcal{X}}^{j}\right\| .
\end{aligned}
$$

Therefore, if $\rho_{j} K_{j} \rightarrow 0$, then

$$
\left\|\nabla_{u}^{g_{j}} \mathcal{X}^{j}-\int_{B\left(x, \rho_{j}\right)} P_{y}^{x}\left(\nabla_{U}^{g_{j}} \overline{\mathcal{X}}^{j}\right) \Psi_{\rho_{j}}(x, y) d \mu_{x}\right\| \leq K_{j} \rho_{j}\left\|\overline{\mathcal{X}}^{j}\right\| \rightarrow 0,
$$

so that we choose very small $\rho_{j}$ such that $\rho_{j} K_{j} \rightarrow 0$. From $\left(\Phi_{k}^{j}\right)^{*} g_{j} \rightarrow \boldsymbol{g}^{k}$ in the $C^{1, \alpha}$-topology and (12), (15), we have

$$
\begin{aligned}
& \left\|\nabla_{u}^{g_{j}} \mathcal{X}^{j}-\nabla_{u}^{g_{j}}\left(\Phi_{k}^{j}\right)_{*}\left(X_{k}\right)\right\|_{C^{0}} \\
& \quad \leq\left\|\nabla_{u}^{g_{j}} \mathcal{X}^{j}-\nabla_{u}^{g_{j}} \overline{\mathcal{X}}^{j}\right\|_{C^{0}}+\left\|\nabla_{u}^{g_{j}} \overline{\mathcal{X}}^{j}-\nabla_{u}^{g_{j}}\left(\Phi_{k}^{j}\right)_{*}\left(X_{k}\right)\right\|_{C^{0}} \rightarrow 0 .
\end{aligned}
$$

From (16), $L_{X_{k}} \mathbf{g}^{k}=0$ and $\left(\Phi_{k}^{j}\right)^{*} g_{j} \rightarrow \boldsymbol{g}^{k}$,

$$
\begin{aligned}
&\left|g_{j}\left(\nabla_{V}^{g_{j}} \mathcal{X}^{j}, W\right)+g_{j}\left(\nabla_{W}^{g_{j}} \mathcal{X}^{j}, V\right)\right| \\
& \leq \mid\left|\boldsymbol{g}^{k}\left(\nabla_{\tilde{V}}^{\mathbf{g}^{k}} X_{k}, \tilde{W}\right)+\boldsymbol{g}^{k}\left(\nabla_{\tilde{W}}^{\boldsymbol{g}^{k}} X_{k}, \tilde{V}\right)\right| \\
&+\left|g_{j}\left(\nabla_{V}^{g_{j}} \mathcal{X}^{j}, W\right)-g_{j}\left(\nabla_{V}^{g_{j}}\left(\Phi_{k}^{j}\right)_{*}\left(X_{k}\right), W\right)\right| \\
&+\left|\boldsymbol{g}^{k}\left(\nabla_{V}^{\mathbf{g}^{k}} X_{k}, W\right)-g_{j}\left(\nabla_{V}^{g_{j}}\left(\Phi_{k}^{j}\right)_{*}\left(X_{k}\right), W\right)\right| \\
&+\left|g_{j}\left(\nabla_{W}^{g_{j}} \mathcal{X}^{j}, V\right)-g_{j}\left(\nabla_{W}^{g_{j}}\left(\Phi_{k}^{j}\right)_{*}\left(X_{k}\right), V\right)\right| \\
&+\left|\boldsymbol{g}^{k}\left(\nabla_{W}^{\mathbf{g}^{k}} X_{k}, V\right)-g_{j}\left(\nabla_{W}^{g_{j}}\left(\Phi_{k}^{j}\right)_{*}\left(X_{k}\right), V\right)\right| \rightarrow 0 .
\end{aligned}
$$

As $j \rightarrow \infty$, we have

$$
\left|L_{\mathcal{X}^{j}} g_{j}(V, W)\right|=\left|g_{j}\left(\nabla_{V}^{g_{j}} \mathcal{X}^{j}, W\right)+g_{j}\left(\nabla_{W}^{g_{j}} \mathcal{X}^{j}, V\right)\right| \rightarrow 0 .
$$

Now we use the integral version of Bochner's formula [3]: As $\mathcal{X}^{j}$ is a $C^{1}$-vector field on $M_{j}$,

$$
\int_{M_{j}}\left\{\operatorname{Ric}_{M_{j}}\left(\mathcal{X}^{j}, \mathcal{X}^{j}\right)+(1 / 2)\left|L_{\mathcal{X}^{j}} g_{j}\right|^{2}-\left|\nabla \mathcal{X}^{j}\right|^{2}-\left(\operatorname{div} \mathcal{X}^{j}\right)^{2}\right\} d v=0
$$


As $L_{\mathcal{X}^{j}} g_{j} \rightarrow 0$ and $\left\|\nabla^{g_{j}} \mathcal{X}^{j}\right\|$ is bounded by virtue of the boundedness of $\left\|\nabla^{\mathbf{g}^{k}} X_{k}\right\|$, we have that

$$
\begin{aligned}
\frac{1}{\operatorname{vol}\left(M_{j}\right)} \int_{M_{j}}\left\{-\operatorname{Ric}_{M_{j}}\left(\mathcal{X}^{j}, \mathcal{X}^{j}\right)+\left|\nabla^{g_{j}} \mathcal{X}^{j}\right|^{2}+\left(\operatorname{div} \mathcal{X}^{j}\right)^{2}\right\} d v \\
=\frac{1}{\operatorname{vol}\left(M_{j}\right)} \int_{M_{j}}\left\{K\left\|\mathcal{X}^{j}\right\|^{2}+\left|\nabla^{g_{j}} \mathcal{X}^{j}\right|^{2}+\left(\operatorname{div} \mathcal{X}^{j}\right)^{2}\right\} d v \\
\quad-\frac{1}{\operatorname{vol}\left(M_{j}\right)} \int_{M_{j}} \operatorname{Ric}_{M_{j}}\left(\mathcal{X}^{j}, \mathcal{X}^{j}\right)+K\left\|\mathcal{X}^{j}\right\|^{2} d v \\
=\frac{1}{\operatorname{vol}\left(M_{j}\right)} \int_{M_{j}} \frac{1}{2}\left|L_{\mathcal{X}^{j}} g_{j}\right|^{2} d v \rightarrow 0 .
\end{aligned}
$$

From (5), $\int_{M_{j}} \operatorname{Ric}_{M_{j}}\left(\mathcal{X}^{j}, \mathcal{X}^{j}\right)+K\left\|\mathcal{X}^{j}\right\|^{2} d v / \operatorname{vol}\left(M_{j}\right) \leq 2 A^{2} \overline{\mathcal{R}}_{M_{j}}(K) \rightarrow 0$. For some coordinate $\Phi_{k}^{j}, \operatorname{vol}\left(\operatorname{Im}\left(\Phi_{k}^{j}\right)\right) \geq \operatorname{vol}\left(M_{j}\right) / C_{0}$. Taking a subsequence, we have

$$
\frac{1}{\operatorname{vol}\left(\operatorname{Im}\left(\Phi_{k}^{j}\right)\right)} \int_{\operatorname{Im}\left(\Phi_{k}^{j}\right)}\left\|\mathcal{X}^{j}\right\|^{2} d v \rightarrow 0
$$

which implies $\mathcal{X}^{j} \rightarrow 0$ almost everywhere in $\operatorname{Im}\left(\Phi_{k}^{j}\right)$. As $\left\|X_{k}-\mathcal{X}^{j}\right\| \rightarrow 0$, we obtain that $X_{k}=0$, which is a contradiction to Lemma 1 . Hence, $\left|\operatorname{Isom}\left(M_{j}\right)\right|$ is bounded for any sequence $\left\{M_{j}\right\}$, which completes the proof of Theorem 2 .

4. Proof of Lemma 1: existence of Killing vector fields. Assume that there exists a sequence of manifolds $M_{j}$ such that $\left|\operatorname{Isom}\left(M_{j}\right)\right| \rightarrow \infty$. Let $M_{j}$ converge to a compact length space $M_{0}$ in the Gromov-Hausdorff metric. We may assume that $\left\{p_{k} \mid k=1, \ldots, N\right\}$ in $M_{0}$ satisfy that $M_{0}$ can be covered by $\bigcup_{i=1}^{N(\varepsilon)} B\left(p_{k}, \varepsilon\right)$ and $B\left(p_{k}, \varepsilon / 2\right)$ are pairwise disjoint. Let $\left\{p_{k}^{j} \mid k=1, \ldots, N(\varepsilon)\right\} \subset M_{j}$ converges to $\left\{p_{k} \mid k=1, \ldots, N(\varepsilon)\right\} \in M_{0}$. We define $F(\phi)(i)$ as the smallest $k$ such that $\phi\left(p_{k}^{j}\right) \in B\left(p_{i}^{j}, \varepsilon\right)$. Then $F$ is a map from $\operatorname{Isom}\left(M_{j}\right)$ to $S(\varepsilon)^{S(\varepsilon)}=\{f \mid f: S(\varepsilon) \rightarrow S(\varepsilon)\}$, where $S(\varepsilon)=\{1, \ldots, N(\varepsilon)\}$. As $\left|S(\varepsilon)^{S(\varepsilon)}\right|=N(\varepsilon)^{N(\varepsilon)}$ and $\left|\operatorname{Isom}\left(M_{j}\right)\right| \rightarrow \infty$, there exists $\phi \in \operatorname{Isom}\left(M_{j}\right)$ such that $\max _{x}\left\{d(\phi(x), x) \mid x \in M_{j}\right\} \leq$ $10 \varepsilon$. Furthermore, we obtain that

$$
|\{\phi \mid \max d(\phi(x), x) \leq 10 \varepsilon\}| \geq \operatorname{Isom}\left(M_{j}\right) / N(\varepsilon)^{N(\varepsilon)} \rightarrow \infty
$$

as $j \rightarrow \infty$.

Let $F_{j}=\left\{\phi_{j, l} \mid l=1, \ldots, n(j)\right\}$ be a set of isometries of $M_{j}$ such that $\max _{x}\left\{d\left(\phi_{j, l}(x)\right.\right.$, $x)\} \leq \varepsilon_{j}$. From (19), we can find a sequence $\varepsilon_{j}$ such that $\varepsilon_{j} \rightarrow 0$ and $\left|F_{j}\right| \rightarrow \infty$ as $j \rightarrow \infty$. If we lift the isometry $\phi_{j, l} \in \operatorname{Isom}\left(M_{j}\right)$ to the isometry $\tilde{\phi}_{j, l}$ on $B(0, r / 100)_{\left(\Phi_{k}^{j}\right)^{*} g_{j}}$ and rescale the metric by multiplying $\varepsilon_{j}^{-2}$ as the proof of Theorem 1 , we have $\| \tilde{\phi}_{j, l}(x)-$ $\left(b_{j, l}^{k}+A_{j, l}^{k} x\right) \|_{C^{2}} \rightarrow 0$ as $j \rightarrow \infty$ for $A_{j, l}^{k} \in O(n, \boldsymbol{R})$ by the same reason as in Theorem 1 . As $O(n, \boldsymbol{R})^{C_{0}}=O(n, \boldsymbol{R}) \times \cdots \times O(n, \boldsymbol{R})$ is compact, there exist $s, t$ such that $\left\|A_{j, s}^{k}-A_{j, t}^{k}\right\| \rightarrow 0$ as $j \rightarrow \infty$ for all $k$, where $C_{0}$ is a number of coverings by $r / 100$-ball in Section 3 . Then 
$\left|\tilde{\phi}_{j, s}^{-1} \tilde{\phi}_{j, t}\right| \rightarrow \infty$. Hence, there exists $\tilde{\phi}_{j}$ such that $\left|\tilde{\phi}_{j}\right| \rightarrow \infty$ and

$$
\left\|\tilde{\phi}_{j}(x)-\left(A_{j} x+b_{j}\right)\right\|_{C^{2}} \rightarrow 0
$$

on $B(0, r)$ with $A_{j} \rightarrow I$, with respect to the rescaled metrics. We use the same arguments as in Theorem 1. We have the convergence

$$
\left(B(0, r / 100),\left\langle\tilde{\phi}_{j}\right\rangle\right)_{\left(\Phi_{k}^{j}\right)^{*} g_{j}} \rightarrow(B(0, r / 100), Z)_{\boldsymbol{g}^{k}}
$$

for isometry group $Z$. By the same reason as in Theorem $1, Z$ is non-trivial. For nontrivial isometry $\gamma \in Z$, we can construct isometries $\gamma_{m}$ such that $d(\gamma(p), p) /(m+2)<$ $d\left(\gamma_{m}(p), p\right)<d(\gamma(p), p) /(m+1)$. Hence, $|Z|=\infty$. Furthermore, there exists a sequence of $\left\{f_{i} \in Z\right\}$ such that $f_{i}$ converges to a translational isometry in the $C^{2}$-topology by (20).

Now we show that there exists a Killing vector field on $B\left(p, \varepsilon_{0} / 100\right)$ in a similar way as in [7]. First, we construct a one-parameter sub(pseudo)group of $Z$ as follows. Let $f_{i} \in Z$ be a sequence such that $f_{i}$ converge to the identity in the $C^{2}$-topology and $\varepsilon_{i}=$ $\max \left\{d\left(f_{i}(x), x\right) \mid x \in B(0, r / 100)_{\left(\Phi_{k}^{j}\right)^{*} g_{j}}\right\} \rightarrow 0$. If $h_{i}=\left[1 / \varepsilon_{i}\right]$, then $f_{i}^{h(i)} \rightarrow f$ for an isometry $f$ by considering a subsequence. By the same reason as in [7], $\lim _{i \rightarrow \infty} f_{i}^{[r h(i)]}$ exists for every $r \in[0,1]$. We denote $\lim _{i \rightarrow \infty} f_{i}^{[r h(i)]}$ by $f(r)$. Then we have $f\left(r_{1}+r_{2}\right)=f\left(r_{1}\right) f\left(r_{2}\right)$ if $r_{1}+r_{2}<r / 2$, so that $f(r)$ is a one-parameter pseudo subgroup of $Z$. As $f(r)$ is an isometry for all $r,\|f(r)\|_{C^{2}} \leq C(r)$ for a function $C$ depending on $r$ (see [2]). It yields a one-parameter (pseudo)subgroup on $B(0, r / 2)_{\left(\Phi_{k}^{j}\right)^{*} g_{j}}$ by $f(t) x$. Then we have a Killing vector field $V$ as follows:

$$
V(x)=\frac{d}{d t} f(t) x=\lim _{h \rightarrow 0} \frac{f(t) \circ f(h) x-f(t) x}{h}=f(t)_{*} \lim _{h \rightarrow 0} \frac{f(h) x-x}{h} .
$$

Taking a subsequence if necessary, $\lim _{t \rightarrow 0}(f(t) x-x) / t$ exists. Now we show that $V$ is a $C^{1}$-vector field. As $f(r)$ is bounded in the $C^{2}$-topology,

$$
\begin{aligned}
\frac{d}{d s} V(\gamma(s)) & =\frac{d}{d s}\left(\frac{d}{d t} f(t)(\gamma(s))\right)=\frac{d}{d t}\left(\frac{d}{d s} f(t)(\gamma(s))=\frac{d}{d t}\left(f(t)_{*}(w)\right)\right) \\
& =\lim _{h \rightarrow 0} \frac{f(t+h)_{*}(w)-f(t)_{*}(w)}{h} \\
& =f(t)_{*}\left(\lim _{h \rightarrow 0} \frac{f(h)_{*}(w)-w}{h}\right)
\end{aligned}
$$

for $\gamma^{\prime}(0)=w$. Hence, there exists a $C^{1}$-Killing vector field on $B(0, r / 2){ }_{\left(\Phi_{k}^{j}\right)^{*} g_{j}}$.

ACKNOWLEDGMENTS. The author would like to thank the referee for his valuable comments. The work was supported by the Korea Research Foundation Grant (KRF-2004-003-C00025).

\section{REFERENCES}

[1] M. T. ANDERSON, Convergence and rigidity of manifolds under Ricci curvature bounds, Invent. Math. 102 (1990), 429-445.

[2] E. Calabi and P. Hartman, On the smoothness of isometries, Duke Math. J. 37 (1970), 741-750.

[ 3 ] X. DAI, Z. Shen AND G. WeI, Negative Ricci curvature and isometry group, Duke Math. J. 76 (1994), 59-73. 
[ 4 ] K. FUKAYA AND T. YAmaguchi, The fundamental groups of almost non-negatively curved manifolds, Ann. of Math. (2) 136 (1992), 253-333.

[ 5 ] M. Gromov, Almost flat manifolds, J. Differential Geom. 13 (1978), 231-241.

[ 6 ] K. Grove And K. Shiohama, A generalized sphere theorem, Ann. of Math. (2) 106 (1977), $201-211$.

[ 7 ] D. Montgomery And L. Zippin, Topological transformation groups, Interscience Publishers, New York, 1955.

[ 8 ] S.-H. PAENG, A generalized almost flat manifold under a bounded weak $C^{0, \alpha}$-norm, Arch. Math. (Basel) 77 (2001), 423-429.

[ 9 ] P. Petersen, G. Wei And R. Ye, Controlled geometry via smoothing, Comment. Math. Helv. 74 (1999), 345-363.

[10] P. Petersen And G. Wei, Relative volume comparison with integral curvature bounds, Geom. Funct. Anal. (1997), 1031-1045.

[11] P. Petersen, Riemannian Geometry, Grad. Texts in Math. 171, Springer, New York, 1998.

[12] X. RonG, A Bochner theorem and applications, Duke Math. J. 91 (1998), 381-392.

[13] T. YAmAGUCHI, The isometry groups of manifolds of nonpositive curvature with finite volume, Math. Z. 189 (1985), 185-192.

DEPARTMENT OF MATHEMATICS

KONKUK UNIVERSITY

1 HWAYANG-DONG, GWANGJIN-GU

SEOUL 143-701

KOREA

E-mail address: shpaeng@konkuk.ac.kr 